\title{
Wind tunnel study on the influence of different parapets on the roof pressure distribution of low-rise buildings
}

\author{
S. Pindado, J. Meseguer
}

\begin{abstract}
High suction loads appear on roofs of low-height buildings. The use of parapets with appropriate height at the roof edges alleviates these loads. The performance of six parapet configurations to decrease the suction loads induced on roofs by oblique winds has been studied in a low speed wind tunnel. The studied parapet configurations include vertical wall parapets, either solid or porous, and cantilevered parapets formed by a small horizontal roof close to the building roof. Low-height parapets with a medium porosity and cantilevered parapets are more efficient than solid parapets to reduce the wind suctions generated on the roofs by conical vortices.
\end{abstract}

\section{Introduction}

In the sharp edges on bluff bodies like low rise buildings, the severe adverse pressure gradients appearing downstream the roof edges frequently cause the boundary layer around the bodies to separate, and generate a vortex flow pattern which produces severe suction loading in the separated flow region.

This phenomenon becomes remarkable in the case of low-rise buildings with incident wind at oblique angles to the edges where the existence of such vortex pattern in the region of the windward facing corner is well established at both model 
scale and full scale. These vortices (also known as delta wing vortices because of their similarity with the vortex generated at the leading edge of delta type wings $[1,2])$ produce a potentially damaging high suction on the roof surface. The highest suction forces appear close to the eaves and the roof edges, and are obtained for oblique wind directions. These severe mean suction peaks, which can produce cladding failures, usually only affect a small roof area near the windward corner, the reason being that the absolute value of the pressure coefficient seems to decrease as the inverse of the root of the distance to the roof corner $[3,4]$.

The use of vertical parapets located at the roofs edges has been studied in the past in order to reduce the high suction caused by the conical vortices [5-9]. As far as we know, most of the studies devoted to the effect of parapets on roof suction loads are dealing with solid parapets, and none or little effort has been dedicated to porous parapets. In spite of that, porous fences are widely used for wind protection in many technical applications, ranging from agriculture to pedestrian comfort.

In the present work the effects of porous parapets on the wind induced pressure distribution on low flat roofs are analysed using wind tunnel testing. In addition, other non-standard parapet configuration (cantilevered parapets) has been tested to get additional insight on the effect of such devices on the pressure distribution on the roofs.

\section{Experimental configuration}

The testing model represents a low-rise building. It is a half-cube $0.32 \mathrm{~m}$ wide, $0.32 \mathrm{~m}$ depth and $0.16 \mathrm{~m}$ high surrounded by $2.5 \mathrm{~mm}$ thick external walls, which grip the different parapets tested (Fig. 1). Therefore, taking into account both the

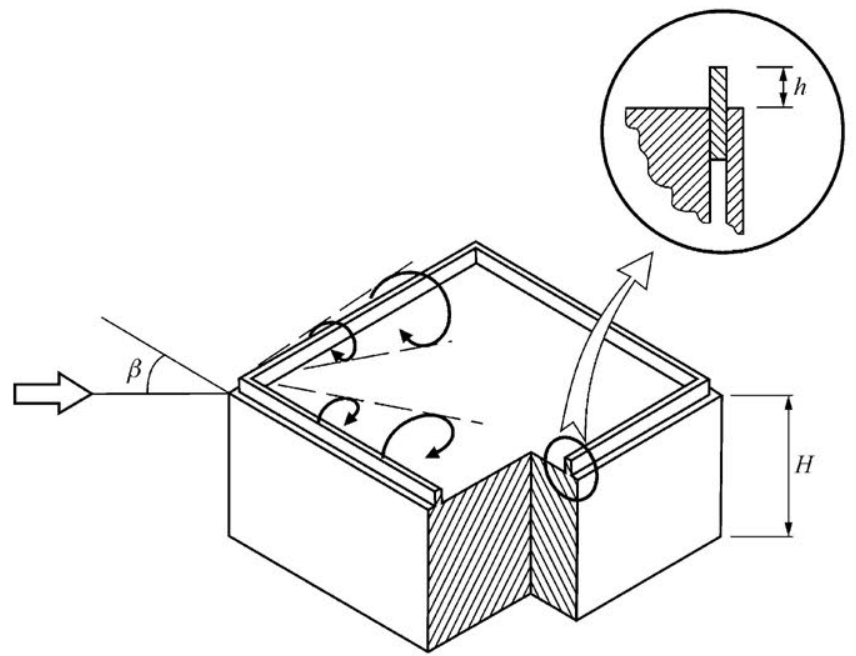

Fig. 1. Sketch of the experimental configuration: a block-type-shaped model with pressure taps on the roof and parapets whose height, $h$, can be modified according to experimental requirements. 


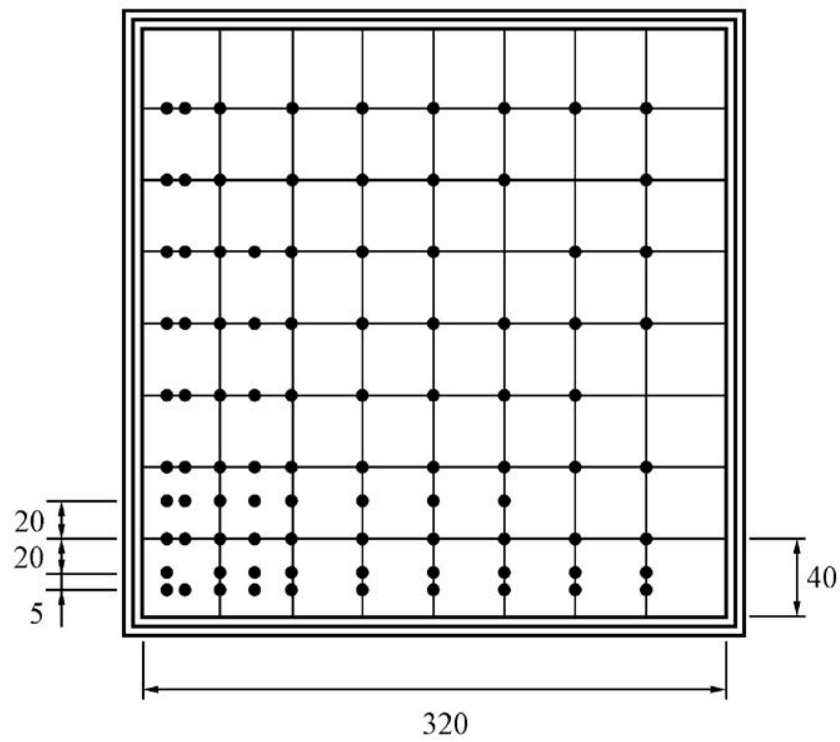

Fig. 2. Arrangement of the pressure taps on the model roof. All dimensions are in millimetres.

thickness of the auxiliary external walls and the parapet thickness, the characteristic length of the roof (the side length) varies between 0.327 and $0.330 \mathrm{~m}$. In all the cases the model has been tested with their windward walls forming an angle $\beta=45^{\circ}$ with respect to the incident wind. This wind direction has been proved to represent the worst case in terms of suction on the roof according to some previous experiments. Ninety-two pressure taps were installed on the roof of the model, arranged as shown in Fig. 2. Each pressure tap consists of a brass tube, $1 \mathrm{~mm}$ inner diameter, which is connected to the pressure measurement instrument by a plastic tube with $1 \mathrm{~mm}$ inner diameter. Plastic tubes are connected to two 48-positions pressure scanners from Scanivalve Corp., each one equipped with a Druck PDCR22 differential pressure transducer. Transducer outputs were sampled at $20 \mathrm{~Hz}$ during $12.5 \mathrm{~s}$ for each measurement. Pressure measurements have been made dimensionless by using the values of both the static pressure and the dynamic pressure of the incident wind at the model roof height. Therefore, the pressure coefficient is defined as usual, $c_{p}=$ $\left(p-p_{\infty}\right) / q_{\infty}$, where $p$ is the mean pressure measured on each tap, and $p_{\infty}$ and $q_{\infty}$ are the static and dynamic pressures upstream the testing model, respectively.

The different parapet types considered in this work are defined in Figs. 3 and 4. The location of parapets on the roof of the testing model is sketched in Fig. 1. Type A parapets are solid plates $2.5 \mathrm{~mm}$ thick. Type B parapet is a commercial square grid made of $1 \mathrm{~mm}$ diameter stainless steel wires with their axes separated $10 \mathrm{~mm}$. The porosity of Type B parapets is $\phi=0.81$. Type C parapets are perforated plates $2.5 \mathrm{~mm}$ thick, with a regular pattern of holes $6 \mathrm{~mm}$ inner diameter whose centres are arranged in a square grid with a $10 \mathrm{~mm}$ side (the porosity of type $\mathrm{C}$ parapets is then 

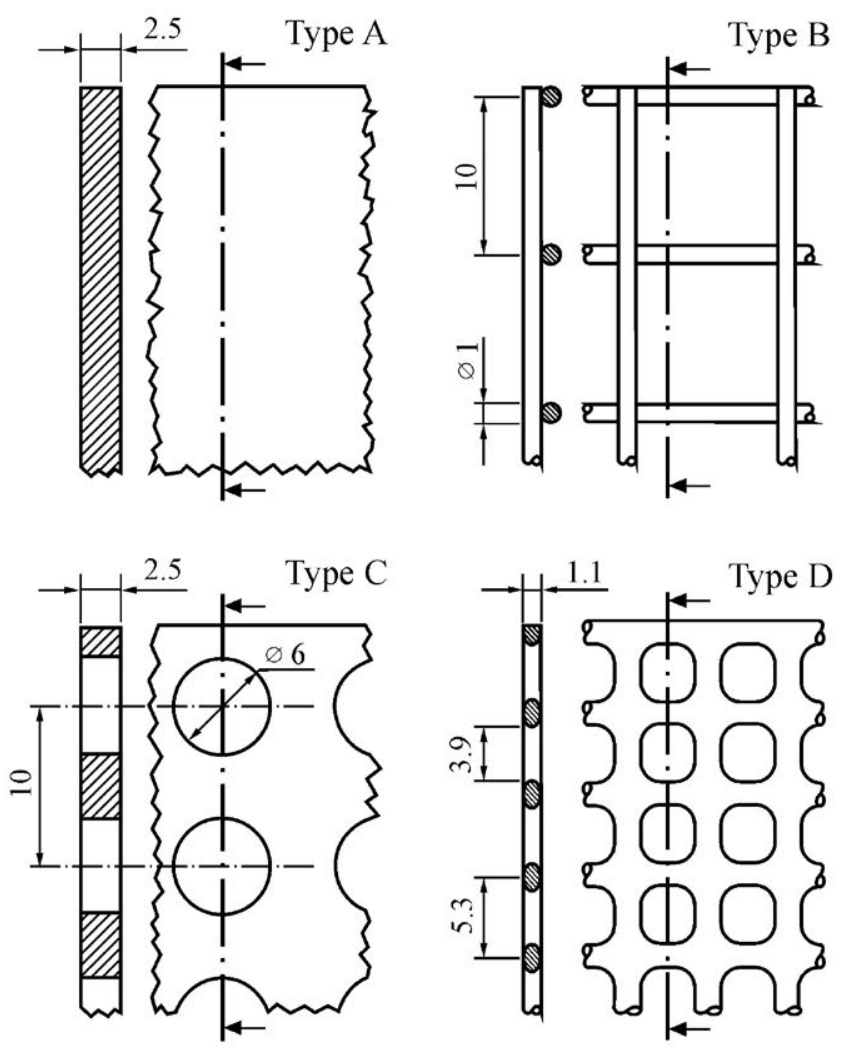

Fig. 3. Sketch of the parapets of type A, B, C and D. All dimensions are in millimetres.

$\phi=0.28$ ). Type D parapet is a commercial plastic grid with a porosity $\phi=0.50$. Finally, type W1 and type W2 parapets consist of small horizontal roofs, $10 \mathrm{~mm}$ width and $1.2 \mathrm{~mm}$ thick, placed over the edges of the model roof (see Fig. 4). The external edges of type $\mathrm{W} 1$ parapets lie $4 \mathrm{~mm}$ outside the roof edges, whereas in type W2 parapets the external edges lie just in the vertical of the roof edges.

Measurements have been carried out in a low velocity wind tunnel at IDR/UPM. The test section is $1.5 \mathrm{~m}$ wide and $1.8 \mathrm{~m}$ high. Taking a $1: 100$ scale for the testing model the wind velocity profile at the model test section was similar to type I atmospheric boundary layer distribution [10]. Two different values of the turbulence intensity of the flow in the test section, at the model roof height have been considered, $I_{u}=2.5 \%$ and $10 \%$. No remarkable differences were observed in the pressure distribution on the model roof although with low-height Type A parapets the highest suction loads increase as the turbulence intensity decreases (see Fig. 5). The wind velocity of the stream at the test section of the wind tunnel, above the boundary layer, was $22 \mathrm{~m} \mathrm{~s}^{-1}$. Additional details on the measurement conditions are available at request to the authors. 

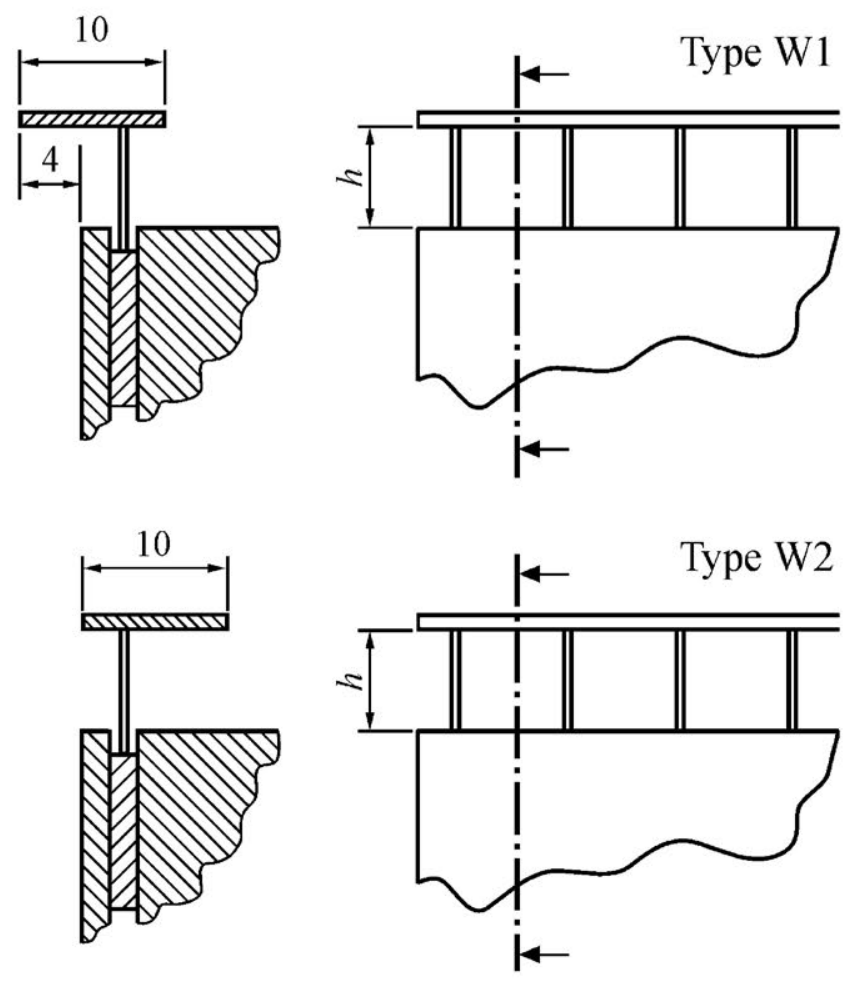

Fig. 4. Sketch of the parapets of type W1 and W2. All dimensions are in millimetres.

\section{Results and discussion}

In Fig. 5 the variation with the relative parapet height, $h / H$, of the minimum mean pressure coefficients measured on the roof is shown. The relative parapet height is defined as the ratio of the parapet height, $h$, to the model height, $H=0.16 \mathrm{~m}$. As it can be observed, the worst measured suction loads are widely affected by the parapet design. In the case of vertical parapets, the effect of the parapet porosity is remarkable, provided the porosity is not large enough ( $\mathrm{C}$ and $\mathrm{D}$ type parapets). This could be explained because of the eddy-turbulence wake generated downwind the parapets which reduces the intensity of the vortex. As it was expected, the suction peak on the roof decreases drastically as the height of the parapet grows.

The very different behaviour shown by type B parapet is due to its very low porosity (type B parapet makes only a tiny perturbation of the flow). Conical vortices are almost unaffected by type B parapets except when one of the horizontal wires forming type B parapet is close enough to the building roof. In this case the turbulent wake produced by one of the mentioned wires, which is parallel to the leading edge, slightly attenuates the conical vortices intensity. 


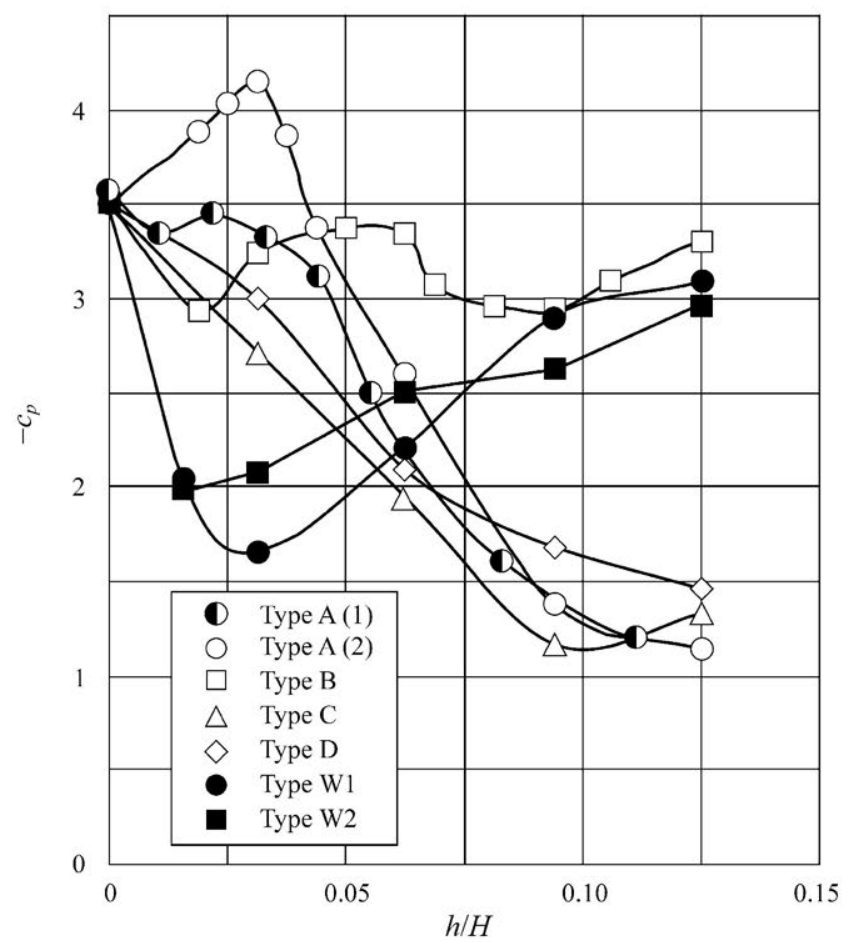

Fig. 5. Variation with the ratio of the parapet height to the model height, $h / H$, of the highest mean suction $-c_{p}$, measured on the model roof for a $45^{\circ}$ incidence wind angle. The symbols identify the parapet type. Type A parapet has been tested at two turbulence intensities, $I_{u}=10 \%$ (1) and $I_{u}=2.5 \%$ (2). All the other parapet types have been tested at $I_{u}=2.5 \%$.

This behaviour can be observed in Fig. 5, where the suction peaks behind type B parapets decrease for relative heights $h / H \approx 0.02$ and 0.08 , which correspond to the configurations where the wire closest to the roof is two to four wire diameters over the roof plane.

Concerning type $\mathrm{W} 1$ and type $\mathrm{W} 2$ parapets, the most remarkable characteristic of these devices is that they seem to be very effective at low relative heights, their performances decreasing as the relative height increases. The reason for this behaviour is that type $\mathrm{W} 1$ and type W2 parapets act like some high lift leading edge devices used in aircraft wings. At low relative heights, there is a high-pressure region before the parapets and a low-pressure region behind them. This pressure gradient forces the injection of a plane jet parallel to the roof surface that sweeps the conical vortex. Note that this effect is more accentuated in the case of W1 type parapets (its leading edge is ahead of the roof edge) than for type W2 parapets (its leading edge is vertically aligned with the roof edge), as one could expect, because in the first case the parapet configuration tend to create a more pronounced high pressure region at the roof leading edge. 


\section{Conclusions}

Experimental data indicate that for low relative height vertical parapets $(h / H<0.05)$, medium porosity parapets are more effective than solid vertical parapets to reduce the suction peaks appearing on the roof, the differences in load reduction between solid and porous parapets being smaller as the relative parapet height grows.

Low-height cantilevered parapets $(h / H<0.031)$ have also produced a very effective reduction of the wind loads on the model roof, even better than vertical parapets (either solid or porous). However, cantilevered parapets present some drawbacks: in addition to a more complex manufacturing, they are poorly effective for high relative parapet heights.

\section{References}

[1] A.M. Mitchell, J. Délery, Research into vortex breakdown control, Prog. Aerospace Sci. 37 (2001) $385-418$.

[2] R.J. Kind, Worst suctions near edges of flat rooftops on low-rise buildings, J. Wind Eng. Ind. Aerodyn. 25 (1986) 31-47.

[3] J.X. Lin, D. Surry, H.W. Tieleman, The distribution of pressure near roof corners of flat roof low buildings, J. Wind Eng. Ind. Aerodyn. 56 (1995) 235-265.

[4] C.W. Letchford, R. Marwood, On the influence of $v$ and $w$ component turbulence on roof pressures beneath conical vortices, J. Wind Eng. Ind. Aerodyn. 69-71 (1997) 567-577.

[5] G. Lythe, D. Surry, Wind loading of flat rooftops with and without parapets, J. Wind Eng. Ind. Aerodyn. 11 (1983) 75-94.

[6] C. Kramer, H.J. Gerhardt, S. Scherer, Wind pressure on block-type buildings, J. Wind Eng. Ind. Aerodyn. 4 (1979) 229-242.

[7] A. Baskaran, T. Stathopoulos, Roof corner wind loads and parapet configurations, J. Wind Eng. Ind. Aerodyn. 29 (1988) 79-88.

[8] T. Stathopoulos, R. Marathe, H. Wu, Mean wind pressures on flat roof corners affected by parapets: field and wind tunnel studies, Eng. Struct. 21 (1999) 629-638.

[9] R.J. Kind, Worst suctions near edges of flat rooftops with parapets, J. Wind Eng. Ind. Aerodyn. 31 (1988) 251-264.

[10] Eurocode 1 (spanish version): Eurocódigo 1, Bases de proyecto y acciones en estructuras. Parte 2-4: Acciones en estructuras. Acciones del viento, UNE-ENV 1991-2-4, AENOR 1998. 